ESJ Humanities

\title{
Pratiques Sociales et Deficit d'Hygiene des Aliments au Sein du Grand Marche de Treichville et le Forum des Marches d'Adjame (Cote d'Ivoire)
}

\author{
Nadège Ahou Kouadio Konan \\ Doctorante en Sociologie de l'Environnement \\ Université Félix Houphouët-Boigny, Côte d'Ivoire
}

Doi:10.19044/esj.2021.v17n9p71

Submitted: 08 February 2021

Accepted: 15 March 2021

Published: 31 March 2021
Copyright 2021 Author(s)

Under Creative Commons BY-NC-ND

4.0 OPEN ACCESS

Cite As:

Kouadio Konan N.A. (2021). Pratiques Sociales et Deficit d'Hygiene des Aliments au Sein du Grand Marche de Treichville et le Forum des Marches d'Adjame (Cote d'Ivoire). European Scientific Journal, ESJ, 17(9), 71. https://doi.org/10.19044/esj.2021.v17n9p71

\section{Résumé}

L'objectif de cet article est d'analyser les pratiques développées par les acteurs qui favorisent le déficit d'hygiène des denrées au sein du Grand marché de Treichville et le Forum des marchés d'Adjamé. Ce mécanisme de dégradation de l'espace environnemental marché se saisit ici à travers leurs comportements et pratiques vis-à-vis du manque de l'hygiène. En adoptant une approche mixte (qualitative et quantitative), l'article s'est appuyé sur la recherche documentaire, l'observation, l'entretien lors de l'enquête en vue de collecter des données, l'administration d'un questionnaire a permis de quantifier les données sur la population cible (commerçants), qui ont servi à rendre compte de la complexité des relations entre hygiène environnementale et hygiène alimentaire, et du danger sanitaire qui en découle. Au terme de notre investigation, les résultats révèlent que l'insalubrité de l'espace environnemental marché dans lequel sont commercialisées les denrées, s'explique par les perceptions que les acteurs mobilisent, et les pratiques qui légitiment leur comportement. La prise de conscience de l'interaction entre l'alimentation, l'environnement et la santé reste un objectif à atteindre.

Mots-clés : Déficit d'Hygiène des Aliments, Insalubrité, Risque Sanitaire, Environnement, Abidjan 


\title{
Social Practices and Food Hygiene Deficit in the Large Treichville Market and the Adjamé Market Forum (Cote d'Ivoire)
}

\author{
Nadège Ahou Kouadio Konan \\ Doctorante en Sociologie de 1'Environnement \\ Université Félix Houphouët-Boigny, Côte d'Ivoire
}

\begin{abstract}
The objective of this article is to analyze the practices developed by the players who favor the food hygiene deficit within the large market of Treichville and the Adjamé markets forum. This mechanism of degradation of the environmental market space is captured here through their behavior and practices vis-à-vis the lack of hygiene. By adopting a mixed approach (qualitative and quantitative), we relied on documentary research, observation, interview and the administration of a questionnaire during the survey in order to collect data that were used to take into account the complexity of the relationship between environmental hygiene and food hygiene as well as the resulting health hazard. At the end of our investigation, the results reveal that the insalubrity of the environmental market space in which foodstuffs are marketed can be explained by the perceptions that stakeholders mobilize, and the practices that legitimize their behavior. Awareness of the interplay between food, environment and health remains a goal.
\end{abstract}

Keywords: Food Hygiene Deficiency, Insalubrity, Health Risk, Environment, Abidjan

\section{Introduction}

Les denrées alimentaires constituent les premiers biens de consommation servant à la satisfaction des besoins primaires de tout être humain (FAO, 2017). Selon la communauté internationale, la qualité des aliments est primordiale pour la sécurité sanitaire des populations (idem). A cet effet, Jeanne Ducatillon (2001) montre l'importance d'une alimentation saine en s'appuyant sur le serment d'Hippocrate, « que ton aliment soit ta seule médecine ». Il fait donc ressortir le lien entre l'alimentation et la santé à travers la qualité des denrées en matière d'hygiène, et l'environnement dans lequel sont commercialisés divers produits alimentaires. La question de l'hygiène pose un problème essentiel tant au niveau local que global. La maîtrise de la sécurité des denrées alimentaires est devenue une préoccupation pour les organisations internationales, les services publics, les consommateurs, la 
grande distribution et les fabricants. C'est un enjeu international de par la mondialisation des marchés, où les consommateurs sont de plus en plus exigeants (Kristina Roesel, 2016). Une défaillance dans le système de consommation des aliments commercialisés à n'importe quelle étape a des conséquences négatives au niveau de la santé publique de la population (Tra Fulbert et Konan Nadège, 2018).

En Côte d'Ivoire, on assiste à une recrudescence de la toxi-infection alimentaire et à l'émergence de certaines maladies. Le pays connait plusieurs épidémiques de choléra en janvier 2011. On dénombrait 933 cas dont 22 décès en septembre 2011. La résurgence de ces maladies est due à l'insalubrité et aux difficultés de gestion des pratiques d'hygiène alimentaires entrainant des risques sanitaires (Rapport de politique national de Santé, 2011). Les données de la Direction d'Hygiène Publique et Santé-Environnement (DHPSE) révèlent des cas d'intoxications alimentaires, dues à la consommation d'aliments avariés ou contaminés en 2018. Des cas ont été signalés également à Kong 2, un village de la sous-préfecture de Yakassé Attobrou, provoqué par la consommation de bouillie de mil, qui a fait 28 victimes dont 12 décès enregistrés, parmi lesquels on dénombre 9 enfants d'âge compris entre 2 et 7 ans (Rapport de DHPSE, 2018). D'autres cas similaires d'intoxications alimentaires ont été constatés également à Bingerville en 2019, dont une trentaine d'élèves pris en charge par les services sanitaires (INHP, 2019). Et pourtant, depuis le 17 octobre 2007, le gouvernement ivoirien déjà avait élaboré un Plan d'Action National de Sécurité Sanitaire des Aliments (PANSSA) en vue d'améliorer la situation de l'hygiène et d'assurer à la population ivoirienne l'accès à une alimentation saine et nutritive (Ministère de la santé, 2010). C'est dans cette optique qu'il s'est engagé à assainir la ville d'Abidjan et sensibiliser les populations. Au niveau local, les mairies disposent d'un service " environnement et hygiène publique », dont la tâche est la lutte contre l'Insalubrité et la sensibilisation des acteurs sur les mesures d'hygiène alimentaires au sein des marchés.

Malgré les actions menées dans le cadre de la sensibilisation, du contrôle et de l'assainissement, l'espace marché rencontre des problèmes en matière d'hygiène. Dans un tel contexte, les denrées commercialisées peuvent constituer un risque pour la sécurité sanitaire des populations. C'est le cas du grand marché de Treichville et le forum des marchés d'Adjamé. Les observations sont perceptibles : l'écoulement continu des eaux usées et la présence des ordures au sein des marchés, l'exposition des denrées à proximité des toilettes publiques mal entretenues ou près des caniveaux à ciel ouvert. Le problème de conservation des aliments commercialisés et des aliments prêts à la consommation exposés à ciel ouvert. Sur la base de ces constats, l'insalubrité de l'espace environnemental marché porte atteinte à la qualité des 
denrées alimentaires commercialisées, entrainant des risques sanitaires des consommateurs.

Au regard de la situation en matière d'hygiène susmentionnée, il est opportun de s'interroger : quelles sont les logiques sociales qui sous-tendent le déficit d'hygiène des aliments au sein des marchés publics ?

De cette question de recherche découlent les interrogations suivantes : Quelles sont les pratiques favorisant le déficit d'hygiène des aliments selon 1'Etat? Quelles sont les pratiques favorisant le déficit d'hygiène des aliments selon les commerçants? Quelles en sont les conséquences sanitaires?

Dès lors, et en considérant que de nombreux travaux mettent en lumière le rapport alimentation, environnement et santé (Traoré, 2007; Tuo, 2010; Tra et Konan, 2018; Brou, 2018), l'objectif de cet article consiste à analyser les comportements des acteurs face au déficit d'hygiène des aliments au sein des grands marchés et des conséquences sanitaires qui en découlent.

\section{Cadre méthodologique}

\subsection{Présentation des sites d'enquête}

L'étude a été réalisée au sein de deux Grands marchés du District d'Abidjan. Le Grand marché de Treichville, situé à l'avenue 02 à quelques mètres du siège de la mairie de Treichville, est compté parmi les grands marchés publics dits modernes du District d'Abidjan. Le Forum des marchés est situé dans la commune d'Adjamé dans le quartier Mairie 1, en bordure du boulevard Nangui Abrogoua, à cent mètres de la mairie et, en face du commissariat du troisième arrondissement. Le choix des localités se justifie par le fait que ces marchés dits moderne construits selon le système Build Operate and Transfer (BOT) sont confrontés à des problèmes d'insalubrité. Notre zone d'étude est représentée sur les cartes (cf carte 1 et 2). 


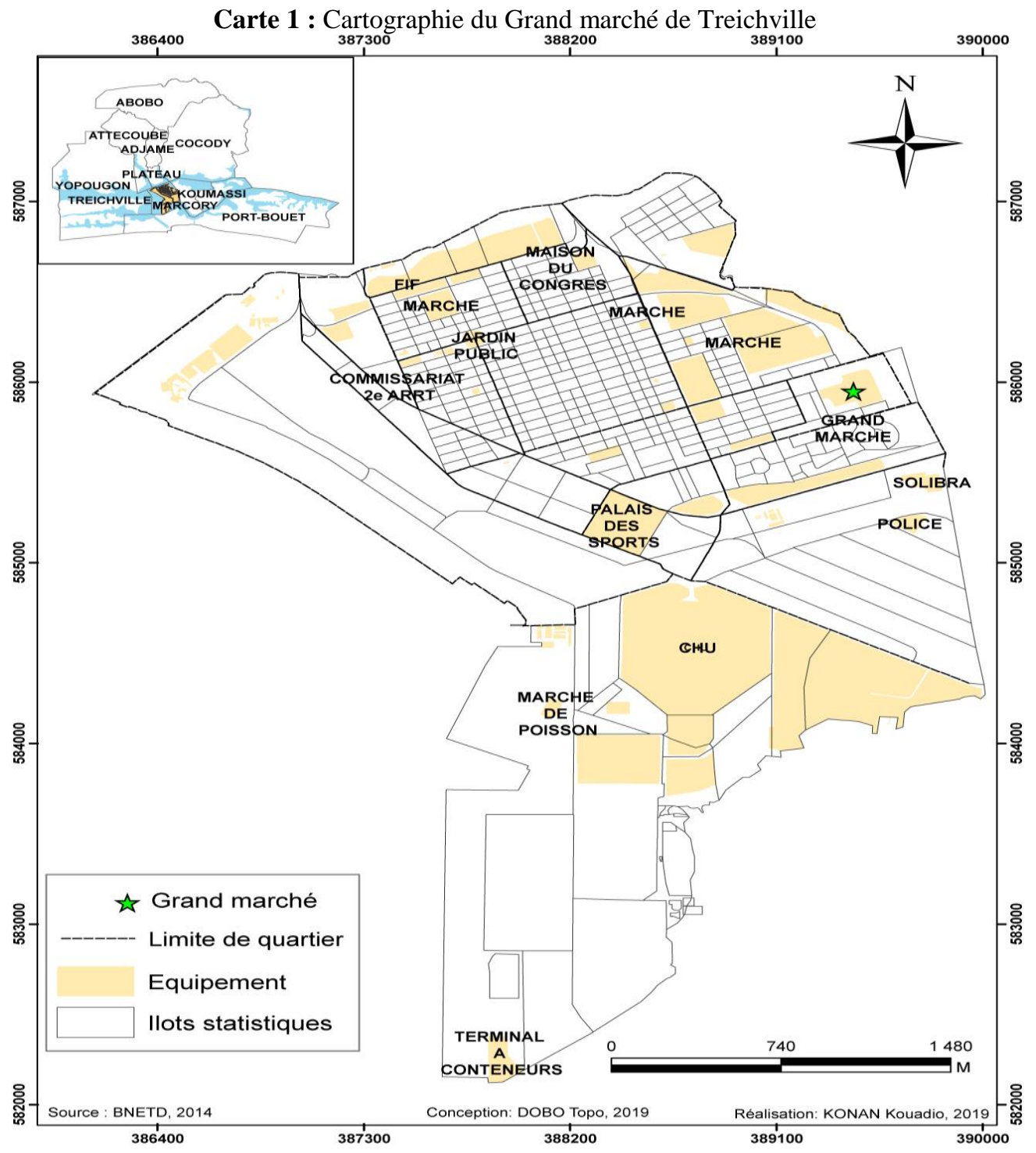


Carte 2 : Cartographie du Forum des marchés d'Adjamé

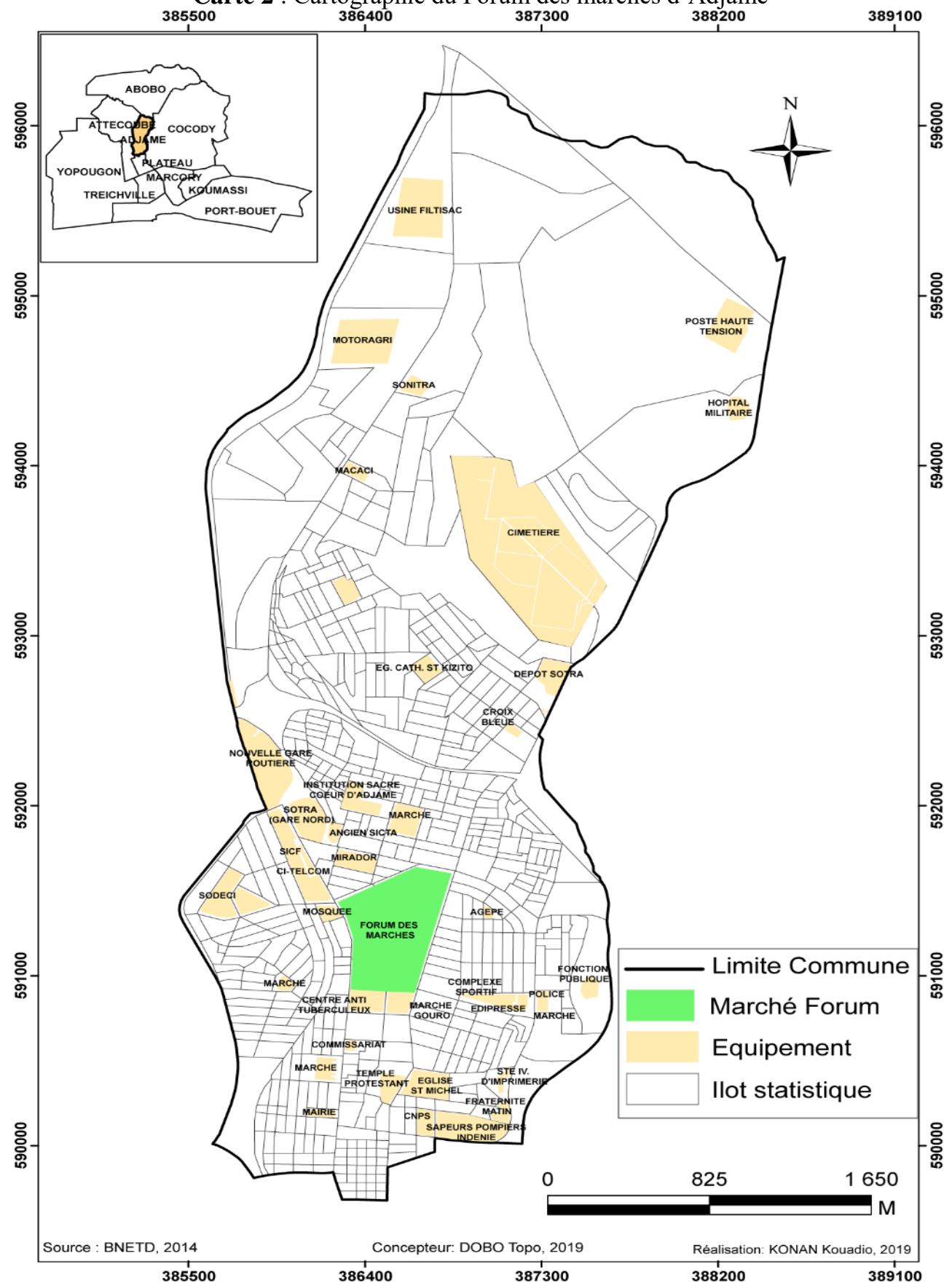

\subsection{Collecte de données et échantillonnage}

En adoptant une méthode mixte, la collecte des informations recueillies en mode qualitatif et quantitatif, a permis d'obtenir des données 
afin d'étudier le phénomène du déficit d'hygiène. Un ensemble de techniques et outils a été ainsi utilisé comme support essentiel dans le recueil des informations. Dans le cadre de la technique de données, il s'agit d'abord de la documentation qui permet de saisir le phénomène du déficit d'hygiène des aliments commercialisés, de l'espace environnemental marché à travers l'exploration des ouvrages en rapport avec la thématique à étudier, et de faire l'état des différents aspects ayant été déjà abordé par d'autres chercheurs ( N'Da Paul, 2006).

Ensuite, l'observation directe a permis de décrire l'état de conservation des denrées. Quant aux entretiens, le type d'entretien utilisé dans cette étude est l'entretien semi directif. Une enquête de terrain a été réalisée auprès des acteurs : commerçants, consommateurs, structures de gestions des marchés et structures étatiques. Comme outils de données, un guide d'entretien est élaboré afin de recueillir des informations en rapport avec leurs perceptions sur la question de l'hygiène des denrées alimentaires. Enfin, le questionnaire permet de quantifier les données sur la population cible (commerçants).

Au cours de l'étude, la technique d'échantillonnage par choix raisonné a été adoptée dans l'approche qualitative pour le choix des enquêtés. Elle consiste pour le chercheur à sélectionner une partie représentative de la population-cible, qui sont des personnes ressources. Nous avons enquêté 31 acteurs cibles. Quant à l'approche quantitative, l'échantillon retenu est un échantillon représentatif selon le calcul de l'échantillon de base à partir de la formule de Fisher (ESSO, 2013). Notre échantillon est basé sur la technique d'échantillonnage aléatoire simple. La taille de l'échantillonnage est 245 commerçants interrogés (115 commerçants au grand marché de Treichville et 130 au forum des marchés).

\subsection{Traitement des Données}

Le traitement de donnée qualitative est fait à l'aide d'un logiciel MAXQDA (Kuckartz Udo, 2014). La méthode d'analyse des données mobilisée est l'analyse du contenu thématique. Cette méthode permet d'analyser le contenu du discours des enquêtés dans le cadre du déficit de l'hygiène des aliments. Quant à l'approche quantitative, le logiciel SPSS 21et Excel adopté pour le traitement des données quantifiables de l'étude pour s'assurer que les informations nécessaires à la réalisation des objectifs sont réellement collectées (Rachad Antonius, 2004). 


\section{Resultats}

\subsection{Les pratiques favorisant le déficit d'hygiène des denrées selon les acteurs étatiques}

\subsubsection{Caractéristiques sociodémographiques des populations}

L'analyse des variables (niveau d'instruction, le sexe, l'anciennement, et l'ethnie) révèlent d'abord que: (77\%) des commerçants sont des analphabètes. ce pourcentage signifie que, plus de la moitié des commerçants que comptent ces différents marchés ne savent ni lire ni écrire. Selon les résultats de l'enquête, on s'aperçoit que les femmes sont majoritaires dans la commercialisation des denrées soit un taux de 71,84\%. Au sein des marchés, on retient également que le grand marché de Treichville et le forum d'Adjamé sont majoritairement occupés par des commerçants étrangers soit un taux 54,69\%. Ces commerçants venant de la CEDEAO préfèrent s'éterniser dans les habitudes et méthodes traditionnelles de vie qu'ils ont acquises par le processus de socialisation depuis le pays d'origine jusqu'au pays d'accueil. Ces commerçants sont en général des personnes adultes. Le nombre d'année d'ancienneté dans le commerce est compris de 10 ans et 30 ans et plus. Il ressort que, les caractéristiques socio démographiques ont permis de comprendre le niveau de connaissance des enquêtés en matière d'hygiène. Ces variables mobilisées, légitiment les pratiques et les comportements des commerçants dans le cadre de la gestion d'hygiène des aliments.

\subsubsection{Surpeuplement des commerçants source d'insalubrité}

Lors des échanges avec les responsables administratifs sur le déficit de l'hygiène de l'espace environnemental marché et les denrées commercialisées. Un enquêté affirme: "Toutes les entrées et même des allées de circulation sont envahies par les vendeurs et par les vendeurs ambulants, il y a plus de commerçants que prévu, c'est les mêmes qui rendent insalubre le marché ». (S.O, Agent mairie du service salubrité et hygiène publique d'Adjamé, entretien, 14.02.2018)

A travers ces propos, la capacité d'accueil de ces marchés est insuffisante à prendre en compte tous les commerçants. Certains s'installent de façon illégale c'est-à-dire n'ayant pas droit à des étals, ni à des box. Ils occupent des endroits stratégiques pour écouler leur marchandise. De fait, le forum des marchés a une capacité de 12000 commerçants. Cependant, celuici abrite actuellement plus de 20000 commerçants. De plus, le grand marché de Treichville ayant une capacité de 5000 commerçants se retrouve à plus de 7000 commerçants prenant en compte le commerce de proximité (données d'enquête, 2018).

Par conséquent, le nombre élevé incontrôlé de commerçants peut favoriser le manque d'hygiène de l'espace environnemental marché. 


\subsubsection{Non-respect des mesures d'hygiène}

Les données recueillies au cours de l'enquête révèlent que des mesures en matière d'hygiène sont établies dans le cadre de la sensibilisation sur la conservation des denrées, de contrôle des denrées, de l'introspection sanitaire, du lavage des marchés après-vente, et ce à partir de 15 heures (données d'enquête, 2018). Toutes ces mesures susmentionnées, ne sont pas prises en considération par les commerçants.

Un agent de la mairie affirme : «quand on vient et on leur parle de la propreté des marchés ou on les sensibilise ces commerçants n'écoutent pas ». (S.K, Responsable du service salubrité et hygiène publique de la mairie de Treichville, entretien, 17.02.2018).

Il ressort de ces propos que les autorités municipales sont confrontées parfois à la réticence des commerçants. Ceux-ci refusent d'appliquer les mesures d'hygiène de l'espace environnemental marché et des denrées commercialisées. Cependant, ces commerçants s'intéressent qu'aux ressources financières. Face à cette situation, le faible niveau d'instruction des commerçants peut être l'une des causes logiques qui peut justifier cette négligence ou ce manque d'intérêt à ne pas respecter les mesures d'hygiène des aliments (Voir graphique ci-dessous).

Graphique : Répartition selon le niveau d'instruction des commerçants

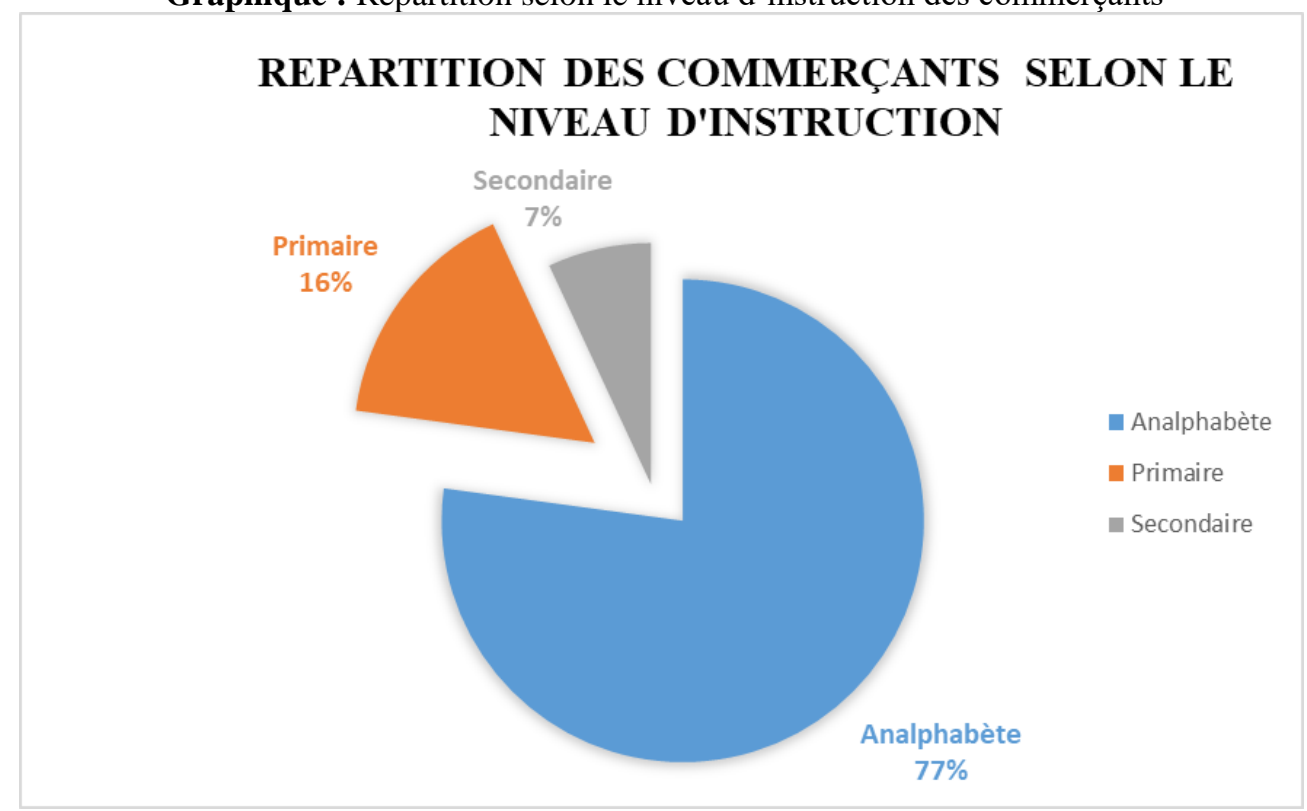

Source : Données d'enquête (2018)

Lors de l'enquête, les personnes interrogées, étant scolarisées ont une faible proportion soit $23 \%$. On remarque une prédominance des enquêtés non scolarisés avec une proportion très élevée soit $77 \%$. On retient que les commerçants qui ont été interrogés sont majoritairement des analphabètes. Ce 
fait justifie l'une des logiques sociales du comportement des commerçants face au non-respect des mesures d'hygiène. Cependant cela peut mettre en cause la sécurité sanitaire des denrées.

\subsection{Pratiques favorisant le déficit d'hygiène selon les commerçants}

\subsubsection{Irrégularité de ramassages des ordures}

Nos enquêtes de terrains ont détecté des irrégularités dans le ramassage des ordures au sein de nos deux marchés. La gestion des ordures n'est donc pas maitrisée (voir tableau ci-dessous).

Tableau : Fréquence de ramassage des ordures par semaine

\begin{tabular}{|ll|l|l|l|l|}
\hline & & Effectifs & Pourcentage & $\begin{array}{l}\text { Pourcentage } \\
\text { valide }\end{array}$ & $\begin{array}{l}\text { Pourcentage } \\
\text { cumulé }\end{array}$ \\
\hline \multirow{4}{*}{ Valide } & 1 & 217 & 88,6 & 88,6 & 88,6 \\
& 2 & 16 & 6,5 & 6,5 & 95,1 \\
& 3 & 12 & 4,9 & 4,9 & 100,0 \\
& Total & 245 & 100,0 & 100,0 & \\
\hline
\end{tabular}

Source : Données d'enquête 2018

Selon les résultats de l'enquête de ce tableau, il ressort que le ramassage des ordures se fait une fois par semaine avec un taux de 88,6\%. Ce manque de responsabilité dans le cadre du ramassage des ordures peut favoriser la dégradation de l'espace environnemental marché. Les commerçants sont exposés à des risques sanitaires dans la mesure où des denrées sont souvent commercialisées à proximité des ordures. Le dysfonctionnement des systèmes de ramassage des ordures, d'assainissement des eaux usées, des eaux pluviales, la collecte et l'élimination des déchets solides rendent ainsi difficile la vie des commerçants en matière d'hygiène (voir photographie 1 et 2). 
Photographie 1 et 2: Quelques images montrant la commercialisation des denrées aux alentours des ordures
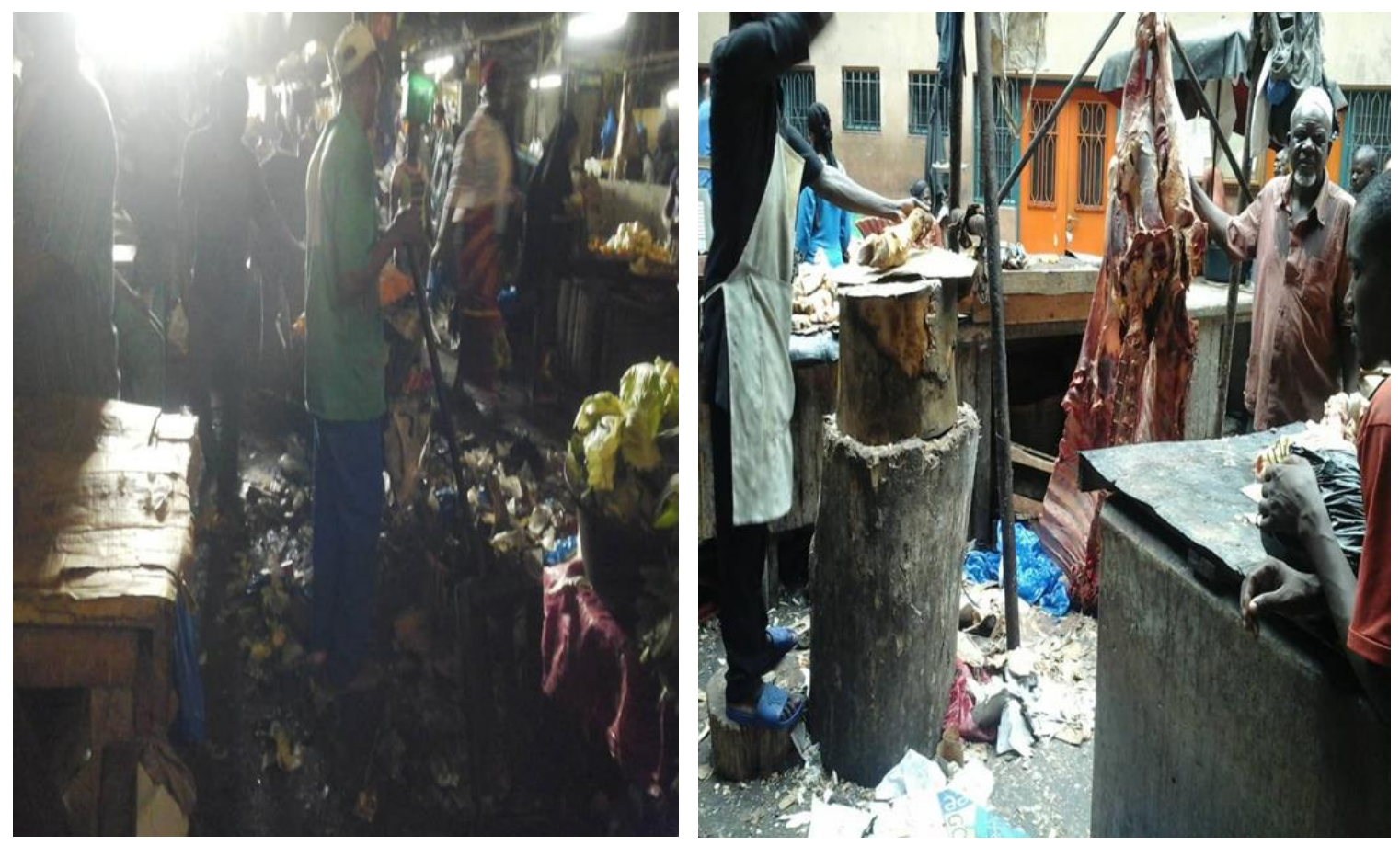

Source : Photo prise par Konan Nadège au Forum des marchés d'Adjamé (2018)

Source : Photo prise par Konan Nadège au Grand marché de Treichville (2018)

\subsubsection{Légitimation des taxes}

Lors des enquêtes, les données révèlent que les autorités sont focalisées sur les ressources financières. La quête du profit est sous-tendue par le fait que les autorités préfèrent faire "'des bénéfices 'malgré l'état d'insalubrité du grand marché de Treichville et le Forum.

C'est en ce sens que certains commerçants affirment : «La mairie ne s'occupe pas de l'entretien du marché. C'est leur affaire de taxe qui les intéressent sinon pour venir ramasser les ordures là faut attendre longtemps dèh.» (J K, boucher, entretien, 19.02.18)

Ces propos justifient le fait que les autorités accordent suffisamment d'intérêt à la recherche de profit sans se soucier véritablement de l'hygiène environnementale des marchés et de la sécurité sanitaire des aliments commercialisés. Au fait, ces autorités se préoccupent de collecter des taxes, qui constituent une des sources des recettes de l'Etat. Ainsi, celles-ci représentent le coût d'un service rendu par la collectivité ou l'organisme qui la 
perçoit. Par conséquent, les rapports existants entre les autorités et les commerçants se situent au niveau des ressources (les taxes).

\subsubsection{Accessibilité des coûts des denrées par les consommateurs}

Pendant les interviews avec les consommateurs, ceux-ci étaient plutôt attirés par le prix abordable des marchandises sans tenir compte de l'hygiène des denrées. En ce sens qu'une consommatrice affirme : «Moi c'est la quantité que je cherche oh surtout qui n'est pas cher, je ne cherche pas la qualité. Si c'est les ordures là où la conservation des aliments là, nous sommes habitués des ordures 》 (B.N, consommateur étudiant titulaire d'une maitrise, entretien, 15.02.18).

Une autre consommatrice affirme: «Ce n'est pas intéressant de vendre autours de la saleté, mais on va faire comment, c'est moins cher pour nous et c'est ce qui nous intéresse» (M. E, professeur, consommatrice, entretien, 15.02.18).

A travers ces propos recueillis, la qualité des denrées semble ne pas être important plus que le besoin de s'approvisionner à tout prix pour nourrir leur famille. Ces propos montrent que les consommateurs ne prennent pas en compte l'hygiène des aliments. Du point de vue des consommateurs, l'expression de l'habitude de vente développée par ces commerçants ne leur permet pas de prendre en considération l'hygiène des aliments. Ces pratiques quotidiennes sont socialement admises par les acteurs.

\subsection{Conséquences liées aux pratiques des acteurs en matière d'hygiène}

Les insuffisances identifiées au cours de l'enquête dans le mode de gestion des aliments commercialisés, ainsi que le cadre environnemental du Forum des marchés d'Adjamé et le Grand marché de Treichville : les ordures ménagères, les eaux usées, les caniveaux à ciel ouvert et la poussière ont des risques sur la santé des consommateurs. Les pratiques des acteurs favorisent l'insalubrité autour des denrées commercialisées. Les marchés rencontrent des difficultés en matière d'hygiène, qui ont des conséquences considérables sur le cadre de vie et la santé des populations. Les égouts et les services sanitaires insuffisants posent également des problèmes d'insalubrité. C'est en cela que le responsable du service hygiène et assainissement affirme :

«La dégradation de l'espace marché entraine des vecteurs de maladies tels que les rongeurs, les cafards, les mouches sont la cause de contamination des denrées commercialisée et peuvent fragiliser la santé des consommateurs. Les porteurs de symptômes visibles: vomissement, diarrhée, jaunisse et fièvre, toux, rhume, plaie septique. Les contaminations de la viande par les bactéries sont les plus fréquentes et les plus connues; il existe aussi des contaminations par des virus et des parasites. L'homme est naturellement 
porteur de microbe et sur toutes les parties de son corps » (L.O, responsable, entretien, 17.02.18)

De ces propos recueillis, les conséquences d'une mauvaise gestion de l'hygiène des denrées et de l'espace environnemental marché exposent les consommateurs à des maladies et des intoxications alimentaires tels que le choléra, la diarrhée, la toux, les vomissements et bien d'autres. Selon ces propos il a été mentionné que l'hygiène corporelle peut contaminer les denrées, et tout peut se transférer sur les aliments à travers la manipulation des mains. Les maladies soulèvent à la fois la question de la sécurité sanitaire des aliments et celle de l'hygiène du travail, qui ne pourront être résolues qu'en améliorant les conditions sanitaires sur les marchés.

A cet effet, les commerçants y compris les consommateurs sont conscients du risque sanitaire des aliments vendus dans un tel environnement. C'est en cela qu'un commerçant du Grand marché de Treichville affirme ceci : «On sait bien qu'on peut tomber malade à cause de la saleté du marché mais on est obligé de vendre, d'exposer nos aliments pour avoir un peu d'argent " (E.K, commerçant, entretien, 15.02.18)

Ces propos traduisent en quelque sorte des risques sanitaires, les intoxications que rencontrent les consommateurs. Mais ces commerçants ont plutôt recours à la recherche des ressources financières sans tenir compte des marchandises de la salubrité des vivres et du cadre environnemental pouvant fragiliser la santé des consommateurs.

\section{Discussion}

\subsection{Pratiques traditionnelles de l'exposition des denrées}

La présente étude, nous a permis de mettre en exergue les pratiques qui favorisent le déficit d'hygiène de l'espace environnemental marché et les denrées. Il semble que cette situation est analogue de certains écrits. La gestion des denrées alimentaires au sein de Forum d'Adjamé et de Grand marché de Treichville est perceptible à travers certaines pratiques. Nous avons entre autre, l'exposition des produits alimentaires à proximité des ordures ménagères et des eaux usées, la vente des aliments prêts à être consommés non loin des toilettes publiques et des caniveaux. Roesel Kristina (2016), à travers son ouvrage intitulé "Sécurité sanitaire des aliments et marchés informels : les produits d'origine animale en Afrique subsaharienne » décrit les modes traditionnelles (manipulations, conservations) des aliments commercialisés, qui sont parfois source de dommages «dangers » pour le consommateur. Ces marchés dits informels échappent aux contrôles sanitaires et de sécurité. Ils ne font que rarement l'objet de taxes et ne sont pas déclarés (Ibid). Les produits vendus sont surtout transformés de manière traditionnelle. Les attitudes orientées vers le profit sont un des risques majeurs pour la sécurité sanitaire des aliments dans les marchés informels. Les aliments sont généralement 
vendus et emballés dans du papier journal d'origine inconnue, qui peut être une source de contamination. Dans cette étude, l'auteur insiste sur les attitudes et le comportement des populations dans l'exercice de la commercialisation des denrées. Cet ouvrage a contribué à montrer l'impact des pratiques d'hygiène sur la santé des consommateurs qui reste une réalité.

Ces résultats rejoignent celui de Konan Nadège (2015), à travers son étude intitulée «Environnement, Santé et Alimentation : étude de cas des pratiques d'hygiène des aliments sur le marché de Wassakara dans la commune de Yopougon ». En effet, l'auteure met en exergue les pratiques des commerçants à travers la commercialisation des aliments. Au fait, ces derniers en étalant ces aliments autours des déchets, posent ainsi d'éventuels problèmes en matière d'hygiène. Pour l'auteure, toutes ces pratiques sont considérées comme des stratégies de vente, un moyen pour permettre aux consommateurs de s'approvisionner.

Ce fait se décrit dans les travaux de Koffi Nevry et Gohou Gaston (2012) à travers son article "Hygiène des aliments et développement soutenable impact du monde invisible (microscopique) sur la réduction de la pauvreté », qui mettent en relief le lien entre la qualité des aliments et le niveau de vie des ménages. Cette qualité maintient les pauvres dans le cercle vicieux de la pauvreté. Il est évident que les modes d'accès à la nourriture tels que le recours aux petits marchés et aux aliments de rue exposent les populations à une nourriture moins saine. Les résultats de son étude confirment l'affluence et les conditions d'insalubrité dans lesquelles les aliments sont vendus. Selon les auteurs, les pratiques d'hygiène précaires favorisent la propagation des maladies. Ces maladies d'origine alimentaire, compagnons privilégiés des endroits sales, sont dues à l'ingestion d'eau ou d'aliments insalubres contaminés par des microorganismes. Dans le cadre de l'étude, cet article a contribué à la compréhension du problème de recherche. Les résultats de cet article ont donné tout le sens théorique et pratique à notre étude. Elle a montré les différents aspects qui peuvent favoriser le déficit d'hygiène des aliments à travers des pratiques des ménages à faibles revenus. En revanche, nous avons orienté notre étude au sein des grands marchés, pour cerner les pratiques, les comportements qui peuvent influencer l'hygiène des aliments commercialisés. La particularité de l'étude, se situe au niveau de la prise en considération de toutes les catégories sociales pour sa faisabilité.

Ces résultats corroborent ceux de Tuo Pega (2010), faisant une analyse critique de la gestion d'hygiène des aliments et du cadre de vie du quartier Williamsville dans la commune d'Adjamé à travers son étude intitulée «Assainissement et gestion de l'environnement dans la commune d'Adjamé ». Cette étude traite des pratiques des populations locales dans le cadre de l'insalubrité des aliments au sein de ce quartier. Selon Tuo Pega (op. cit.), le problème d'hygiène s'aggrave avec le fait que la population elle-même 
jette les déchets dans les caniveaux à ciel ouvert. Le dysfonctionnement des réseaux d'égouts par endroits en ce qui concerne les nuisances au sein de ce quartier. D'ailleurs, l'on rencontre toute sorte de déchets, et non loin desquels on assiste à la vente des aliments tels que la viande, du poisson et des aliments prêts à être consommés. A travers ses investigations, l'auteur stipule que les comportements des populations sont aussi source de pollution, entrainent des nuisances qui favorisent le développement des agents pathogènes depuis quelques années. De cette étude, l'auteur met en évidence le fait que, les comportements des acteurs sont parfois source de pollution dans la mesure où les populations participent à l'insalubrité de ces quartiers. Cependant, il ne nous permet pas de percevoir la dimension idéologique pour comprendre ces comportements.

\subsection{Connaissances des mesures d'hygiène au sein des marchés}

Les commerçants et même les consommateurs n'ont pas suffisamment une éducation des règles élémentaires d'hygiène et cela pose problème de santé publique. Les campagnes de sensibilisation mises en place par les autorités publiques ne sont pas adaptées aux réalités sociales du Grand marché de Treichville et de Forum des marchés d'Adjamé.

Cet argument est partagé par Bian Young (2005). Il présente dans ses travaux les défis mis en place pour la sécurité sanitaire des aliments en Chine. Pour lui, l'hygiène alimentaire a atteint une situation critique devenant préoccupante pour les populations et le gouvernement. Les capacités ainsi que le contrôle des aliments par les organes gouvernementaux, sont respectivement faibles et lent. Les actions prises en matière de sensibilisation doivent être strictes dans l'application (Bian Y, op.cit.). La non- connaissance des règles élémentaires d'hygiène par les acteurs concernés est bien souvent l'une des logiques sociales qui légitime les pratiques des commerçants dans la commercialisation des denrées alimentaires. Ces résultats corroborent celui de Traoré Kassoum (2007), d'Abanou Mathieu (1997), d'Assougba Kablan (2015). Selon ces auteurs, les dysfonctionnements et les insuffisances au niveau des administrations concernés, font face aux comportements et attitudes des populations dans la gestion de l'hygiène publique. Par ailleurs, la prise de conscience de la relation entre les activités de l'homme, son environnement et sa santé reste un objectif à atteindre. En réalité, il faut tenir compte de l'implication des populations, dans les programmes de gestion d'hygiène.

\subsection{Conséquence sanitaire face au déficit d'hygiène des aliments}

Le Grand marché de Treichville et le Forum des marchés sont des endroits où s'effectue l'écoulement final des diverses denrées alimentaires destinées à la consommation. Les comportements et attitudes des 
commerçants en matière d'hygiène des aliments ont des conséquences considérables du cadre de vie et de la santé des consommateurs. Cette assertion est partagée par Alfred Traoré (2002), à travers son étude fait une analyse critique de la salubrité des aliments en Afrique. Selon lui, les maladies d'origine alimentaire constituent un problème de santé publique. Chaque année des personnes souffrent des maladies dues à des aliments contaminés. Le manque d'hygiène affecte l'exposition des aliments de rue. Le risque d'intoxication alimentaire demeure un problème essentiel pour la santé des populations. Cette étude a fait ressortir la relation que la population entretient avec son espace environnemental et son comportement face à la conservation et la manipulation des aliments vendus au sein des quartiers et tient compte du risque sanitaire qui en découle.

Cette idée corrobore celui de Kafando Yamba (2003), dans son ouvrage intitulé «Environnement urbain et problème de Santé à Ouagadougou : cas du quartier Cissin», fait l'état des lieux en mettant l'accent sur l'hygiène des aliments commercialisés sans aucune mesure d'hygiène. Selon lui, les aliments proposés par des restaurants traditionnels laissent cependant à désirer, préparés et vendus dans un souci de gain économique, peu d'attention est accordée à la préparation, l'entretien et à la manipulation des aliments. Le cadre environnemental du lieu de vente pose problème: la proximité des caniveaux, la voirie, les gaz et fumées des pots d'échappement, la poussière ainsi que la présence d'animaux errants, d'insectes, de flaques d'eau et de déchets dans les lieux de vente et de préparation, ne garantissent nullement toutes les conditions d'hygiène alimentaire. Ces modes de gestion peuvent fragiliser la santé des populations.

Ces pratiques inadaptées entrainent des conséquences au niveau de la santé de la population. Par ailleurs, les pratiques quotidiennes en matière d'hygiène dépendent du niveau de conscience des acteurs.

\section{Conclusion}

Affirmer que les pratiques des acteurs favorisent le déficit d'hygiène des denrées semble pertinentes au regard des observations faites sur le Grand marché de Treichville et le Forum des marchés d'Adjamé. L'article met en évidence les différentes formes de pratique autour des denrées face au manque d'hygiène de l'espace marché. Le principe relatif à l'hygiène des aliments et l'espace marché n'est pas pris en compte par les acteurs. Ces pratiques relèvent du mode de vie, des attitudes et comportements face au déficit d'hygiène.

Il faut souligner que ces pratiques quotidiennes sont socialement admises par les acteurs (commerçants et consommateurs). Le manque d'hygiène affecte non seulement ces aliments, mais peut aussi impacter la sécurité sanitaire des consommateurs. Les résultats obtenus rendent compte du caractère insalubre de l'espace environnemental marché dans lequel sont 
commercialisées les denrées. Cependant, l'un des enjeux spécifiques auquel s'intéressent les acteurs (structures étatiques et commerçants) se situe plutôt au niveau des ressources financières. Les mesures d'hygiène des aliments ne sont pas prises en compte par ceux-ci. D'autres perspectives sont ouvertes pour la poursuite de la recherche. Les aspects comme la pauvreté et le genre n'ont pas été étudiées dans l'article, pourrait être une étude sociologique pour élargir des réflexions sur la question d'hygiène des aliments au sein des marchés pour apporter des solutions adéquates.

\section{References:}

1. Abanou Kouakou, M. (1997). Contribution à l'amélioration de l'hygiène environnementale du quartier Yobou Lambert à Treichville, Mémoire de maitrise, Université de Cocody, Abidjan.

2. Assougba, K. (2015). Logiques sociales de la gouvernance des marchés dans la commune d'Adjamé, Thèse unique de doctorat, Université Felix Houphouët-Boigny, Abidjan.

3. Aurélie, P. (2005). Marchés au «nord », marchés au «sud». Visages et situations des marchés dans les villes de Marseille et d'Abidjan. Rives Méditerranéennes, (en ligne), http:/www.sup.adc.Varia.fr/bil/acti/, rtf.

4. Bian, Y. (2005). Les défis de la sécurité alimentaire en chine. Sécurité sanitaire des aliments, 82, 4-14.

5. Brou, N. (2018). Perceptions Sociales De L'hygiène Et De L'assainissement En Milieu Urbain Et Rural Ivoirien. European Scientific Journal, 14(2), 16-18.

6. Ducatillon, J. (2001). Le serment d'Hippocrate, problèmes et interprétations. Bulletin de l'association Guillaume Bude, 1, 34-61.

7. FAO. (2017). L'État de la sécurité alimentaire et de la nutrition dans le monde 2017. Renforcer la résilience pour favoriser la paix et la sécurité alimentaire. Rome, FAO. (en ligne), http:/www.fao.fr/bil/acti/, rtf.

8. INHP. (2019). Rapport du service de surveillance épidémiologique. Abidjan : INHP.

9. Kafando, Y. (2003). Environnement urbain et problème de Santé à Ouagadougou: cas du quartier Cissin. Mémoire de maitrise, Université de Ouagadougou.

10. Koffi, N. et Goho, G. (2012). Hygiène des aliments et développement soutenable: impact du monde invisible (microscopique) sur la réduction de la pauvreté, ISE, MA, ph.D. CESS Institute, Québec, Canada.

11. Konan, N. (2015). Environnement, Santé et Alimentation : étude de cas des pratiques d'hygiène des aliments sur le marché de Wassakara dans 
la commune de Yopougon. Mémoire de Master, Université Felix Houphouët-Boigny, Abidjan.

12. Kuckartz, Udo. (2014). Qualitative Text Analysis. A Guide to Methods, Practice and Using Software. London: SAGE Publications.

13. Ministère de la santé. (2010). Plan national de développement sanitaire 2009-2013 constitution de la République de Côte d'Ivoire du 23 juillet 2000, (en ligne), http:/www.sup.adc.santé.fr/bil/acti/, rtf.

14. N'da, P. (2006). Méthodologie de la recherche: de la problématique à la discussion des résultats, comment réaliser un mémoire, une thèse

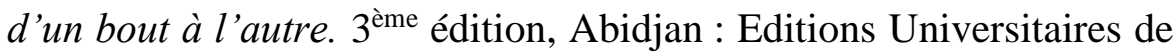
Côte d'Ivoire.

15. Rachad, A. (2004). Initiation au logiciel d'analyse statistique SPSS. Laboratoires et exercices divers. Montréal: département de sociologie, Université du Québec à Montréal, Notes de cours pour le cours SOC 4206. Hiver 2004, 95.

16. Roesel, K. (2016). Sécurité sanitaire des aliments et marchés informels: les produits d'origine animale en Afrique Subsaharienne. Nairobi: Institut International de Recherche sur l'Elevage.

17. Tra, F. et Konan, N. (2018). Environnement et conscience sanitaire des commerçants et consommateurs de Wassakara à Yopougon (Côte d'Ivoire), in European Journal of Scientific Reseach, vol.148, $n^{\circ}$, ISBN : 1450-216X, 307-318.

18. Traoré, A. (2002). Hygiène alimentaire et problématique de l'alimentation de rue en Afrique de l'Ouest. Source de maladies, 6(1), $155-158$.

19. Traoré, K. (2007). De la sensibilisation des populations à la gestion de l'environnement urbain dans les quartiers précaires de la ville d'Abidjan. Étude de la population, 22, 155-160.

20. Tuo, P. 2010. Assainissement et gestion de l'environnement dans la commune d'Adjamé : cas de Williamsville. Mémoire de DEA, Université de Cocody, Abidjan, Côte d'Ivoire. 